
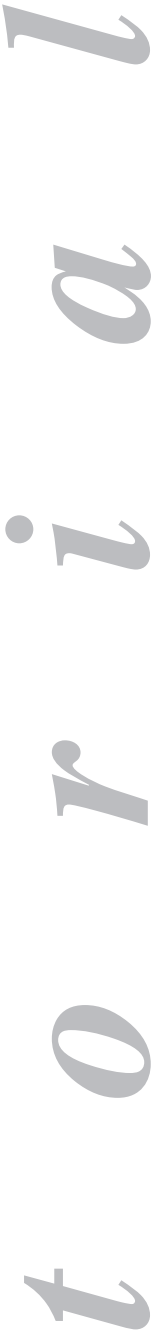

3
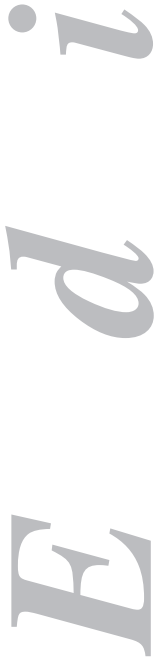

\section{Endoscopic ultrasonography - an established technique with a brilliant future}

Endoscopic ultrasonography (EUS) or echoendoscopy is an imaging technique that emerged over two decades ago, during the previous era of computerized axial tomography (CAT), for improved pancreas examination (1-3). The high resolution of images provided by conventional echoendoscopes has rendered EUS a preferred technique for the study of digestive tumors (1-3). Current tumor diagnostic and staging protocols in most third-level hospitals within our healthcare system include EUS (1-3). Malignancies arising in the esophagus, stomach, rectum, and most particularly the pancreas and bile ducts represent some of the better established indications for EUS. The launch of linear puncture echoendoscopes (EUS-FNP) during the early 1990s was of paramount importance, and brought about new indications for EUS (4). In this issue, two excellent papers on echoendoscopy deserve to be highlighted $(5,6)$.

Iglesias et al. (5) provide a detailed, critical review on the current role of EUS and EUS-FNP in the diagnosis and staging of pancreas cancer, and reach three key conclusions that are discussed below.

Conclusion 1. "EUS is the diagnostic technique of choice for patients suspected of having a pancreatic tumor". Most authors agree with this appraisal, which is reflected in the literature (helical CT vs. EUS: 98 vs. 77\%, p < 0.0001) $(7,8)$. That is, in patients with clinical suspicion of pancreatic cancer and a negative CT scan echoendoscopy would be necessary to exclude malignancy. The superiority of EUS over CAT and MRI is based on the former technique's higher resolution and sensitivity for the diagnosis of tumors $<2 \mathrm{~cm}$ (CAT $v s$. MRI $v s$. EUS: $40,33,90 \%)(9)$.

Conclusion 2. "The accuracy of EUS for the staging of pancreas cancer (7090\%) is equal to or higher than that of other imaging modalities". This statement is more controversial. A systematic review by DeWitt (11 prospective studies, 678 patients) concludes that EUS is superior to multicut helicoidal CT in assessing local/regional tumor extent and splenoportal axis involvement (10). This review found no significant differences in accuracy regarding node staging or overall vascular involvement (including arterial involvement). As a general rule, a pancreatic tumor is usually considered irresectable in the presence of distant metastases or local infiltration of arteries (celiac trunk, hepatic artery, superior mesenteric artery) or veins (extensive infiltration of portal vein or superior mesenteric vein). However, this "theoretical cutoff point" cannot be established so easily in real life. Not uncommonly, a tumor not infiltrating vascular planes cannot be resected during surgery because of a surrounding severe desmoplastic/inflammatory response that prevents tumor-vessel separation. Surgical team experience in this type of procedure and revascularization techniques will greatly 
determine tumor resectability. From a practical standpoint, establishing whether a pancreatic tumor is surgically resectable is an extremely complicated task, even during surgery. Providing surgeons with all sorts of available imaging data (including EUS data) before the procedure should help make the most appropriate decision for each patient. Unfortunately, no single technique alone can ensure resectability.

Conclusion 3. "EUS-FNP has a high diagnostic accuracy (> 90\%) and reduced complication rate $(<1 \%)$ for pancreas cancer". While EUS-FNP has a high yield in this patient population (11), not all authors endorse the same indications. In our view, EUS-FNP should only be used for pancreatic masses when findings may modify patient management. Clinical settings where the use of EUS-FNP may be advocated for include: a) suspicion of tumor histology other than pancreatic ductal adenocarcinoma (lymphoma, neuroendocrine tumor, metastases from other tumors), because of therapeutic and/or prognostic implications may vary; b) irresectable pancreatic tumor according to imaging techniques (not eligible for surgical resection, eligible for chemotherapy); and c) patients with uncertain resectability who will be offered neoadjuvant therapy before the procedure (12). In our opinion a biopsy cannot be warranted for pancreatic lesions consistent with ductal adenocarcinoma that appear to be resectable on imaging tests, since puncture findings (either positive or negative) will not change patient management (attempt at surgical resection), and patients would thus be exposed to unnecessary risks (12).

On the other hand, we should bear in mind that EUS has evolved since inception to become a major therapeutic tool. The ability to perform EUS-FNP with therapeutic linear echoendoscopes fitted with working channels has favored the development of various EUS-guided therapies: celiac plexus neurolysis, pancreatic pseudocyst and perirectal abscess drainage, pancreas and bile-duct puncturing and drainage for tumor palliation, brachytherapy seed implants, etc. (13). Experience in this field is still limited (not available in all centers), and both efficacy and safety are usually poorly understood. This fact has awakened a growing interest in research using animal models to validate the effectiveness and safety of such therapies. A clear example is the original paper by LópezMartín et al. (6). The authors of this study follow the line of investigation opened by Fritscher Ravens (14), and used an animal model to investigate the feasibility of accessing heart chambers using transesophageal EUS-FNP, as well as the procedure's side effects on the heart (arrhythmia, bleeding, etc.). While the study recruited a small number of animals, the authors are first to demonstrate that right heart chambers can be accessed through the interatrial septum with no evidence of complications. However, potential therapies via this novel approach remain to be demonstrated. In our view, the great merit of this study lies in the fact that this is the first report in our setting where an animal model is used for echoendoscopical research. Such pioneering studies in our field open new lines of research into the interesting arena of therapeutic echoendoscopy. The expansion of echoendoscopical research and its use in animal models show that EUS enjoys perfect health in our setting, and that some of the existing teams are highly qualified in this area.

In summary, EUS is a clearly established technique in our healthcare system, and is usually included within the preoperative algorithm for most digestive tumors, among which pancreas carcinoma remains outstanding. The relevant role of EUS in tumor identification, loco-regional spread assessment, and biopsy col- 
lection is widely endorsed by the scientific community. The huge expansion witnessed in the last few years regarding the field of EUS-guided therapy requires prior trials using animal models. The publication in our setting of the first three papers on therapeutic echoendoscopy in animal models is praiseworthy indeed, and a reflection of the perfect health enjoyed by echoendoscopy at present.

\section{E. Vázquez-Sequeiros}

Service of Gastroenterology. University Hospital Ramón y Cajal. Madrid, Spain

\section{REFERENCES}

1. Akahoshi K, Chijiiwa Y, Nakano I, Nawata H, Ogawa Y, Tanaka M, et al. Diagnosis and staging of pancreatic cancer by endoscopic ultrasound. Br J Radiol 1998; 71: 492-6.

2. Palazzo L, Roseau G, Gayet B. Endoscopic ultrasonography in the diagnosis and stating of pancreatic adenocarcinoma. Result of a prospective study with comparison to ultrasonography and CT scan. Endoscopy 1993; 25: $143-50$.

3. Vazquez-Sequeiros E, Wiersema MJ. The role of endoscopic ultrasonography in the diagnosis, staging and management of pancreatic diseases status. Current Gastroenterology Reports 2000; 2: 125-32.

4. Wiersema MJ, Vilmann P, Giovannini M, Chang KJ, Wiersema LM. Endosonography-guided fine-needle aspiration biopsy: diagnostic accuracy and complication assessment. Gastroenterology 1997; 112: 1087-95.

5. Iglesias García J, Lariño Noia J, Domínguez Muñoz E. Endoscopic ultrasounds in the diagnosis and staging of pancreatic cancer. Rev Esp Enferm Dig 2009; 101: 631-8.

6. López Martín A, Pérez-Paredes M, Esteban P, Latorre R, Soria F, Lima R, et al. Transesophageal access to the cardiac cavities and descending thoracic aorta via echoencoscopy. An experimental study. Rev Esp Enferm Dig 2009; 101: 601-9.

7. Howard TJ, Chin AC, Streib EW, et al. Value of helical computed tomography, angiography and endoscopic ultrasound in determining resectability of periampullary carcinoma. Am J Surg 1997; 174: 237-41.

8. Legmann P, Vignaux O, Dousset B, Baraza AJ, Palazzo L, Dumontier I, et al. Pancreatic tumors: comparison of dual phase helical CT and endoscopic sonography. AJR Am J Roentgenol 1998; 170: 1315-22.

9. Müller MF, Meyenberger C, Bertschinger P, Schaer R, Marincek B. Pancreatic tumors: evaluation with endoscopic US, CT, and MR imaging. Radiology 1994; 190: 745-51.

10. DeWitt J, Deveraux BM, Lehman GA, Sherman S, Imperiale TF. Comparison of endoscopic ultrasound and computed tomography for the preoperative evaluation of pancreatic cancer: a systematic review. Clin Gastroenterol Hepatol 2006; 4: 717-25.

11. Eloubeidi MA, Varadarajulu S, Desai S, Shirley R, Heslin MJ, Mehra M, et al. A prospective evaluation of an algorithm incorporating routine preoperative endoscopic ultrasound-guided fine needle aspiration in suspected pancreatic cancer. J Gastrointest Surg 2007; 11(7): 813-9.

12. Varadarajulu S, Wallace MB. Applications of endoscopic ultrasonography in pancreatic cancer. Cancer Control 2004; 11(1): 15-22.

13. Hawes RH, Van Dam J, Varadarajulu S. EUS 2008 Working Group Document: Interventional EUS. A road map for the future. Gastrointest Endosc 2009; 69(2): S1-2.

14. Fritscher-Ravens A, Ganbari A, Mose CA, Swain P, Koehler P, Patel K. Transesophageal endoscopic ultrasound-guided access to the heart. Endoscopy 2007; 39: 385-9. 


\section{Ultrasonografía endoscópica: una técnica consolidada con un brillante futuro}

La ultrasonografía endoscópica o ecoendoscopia (USE) es una técnica de imagen que surgió hace algo más de dos décadas, en la era previa a la tomografía axial computadorizada (TAC), para un mejor estudio del páncreas (1-3). La elevada resolución de las imágenes proporcionadas por los ecoendoscopios convencionales ha permitido situar a esta técnica en un lugar preferente a la hora de estudiar los tumores digestivos (1-3). En la actualidad, los protocolos de diagnóstico y estadificación tumoral de la mayor parte de los hospitales de tercer nivel de nuestro sistema sanitario incluyen la USE (1-3). El cáncer de esófago, estómago, recto y sobre todo páncreas/vía biliar son algunas de las indicaciones más establecidas para la realización de USE. La incorporación del ecoendoscopio lineal de punción (USE-PAAF) a comienzos de los años 90, supuso un avance importantísimo que propició la aparición de nuevas indicaciones para la USE (4). En este número de la revista se presentan dos excelentes trabajos sobre ecoendoscopia que merecen ser resaltados $(5,6)$.

Iglesias y cols. (5), realizan una revisión detallada y crítica sobre el papel actual de la USE y USE-PAAF en el diagnóstico y estadificación del cáncer de páncreas, alcanzando 3 conclusiones fundamentales que se comentan y discuten a continuación.

Conclusión 1. "En pacientes con sospecha de tumor en el páncreas la USE es la técnica diagnóstica de elección". La mayoría de los autores están de acuerdo con esta apreciación y así lo atestigua la literatura existente (TAC helicoidal vs. USE: 98 vs. $77 \%, \mathrm{p}<0,0001)(7,8)$. Es decir, en pacientes con sospecha clínica de cáncer de páncreas en los que el TAC es negativo, sería necesario realizar una ecoendoscopia para descartar la existencia de una lesión maligna. La superioridad de la USE sobre TAC y RMN radica en su mayor capacidad de resolución y su mayor sensibilidad para diagnosticar tumores $<2 \mathrm{~cm}$ (TAC vs. RMN vs. USE: 40, 33, 90\%) (9).

Conclusión 2. "La precisión de la USE para la estadificación del cáncer de páncreas (70-90\%) es igual o superior a la alcanzada por otras técnicas de imagen”. Sobre este aspecto existe mayor controversia. La revisión sistemática publicada por DeWitt (11 estudios prospectivos y 678 pacientes), concluye que la USE es superior al TAC helicoidal muticorte para valorar la extensión local/regional del tumor y la afectación vascular del eje esplenoportal (10). En esta revisión no se encontraron diferencias significativas en la precisión de la estadificación ganglionar o la afectación vascular global (incluyendo afectación arterial). Por regla general, se suele aceptar que un tumor de páncreas es irresecable cuando tiene metástasis a distancia o infiltra localmente los vasos arteriales (tronco celiaco, arteria hepática o mesentérica superior) o venosos (infiltración extensa de vena porta y mesentérica superior). Sin embargo, este "punto de corte teórico" no es tan fácil de establecer en la vida real. No es infrecuente que un tumor que no infiltra los planos vasculares, no pueda ser resecado por el equipo quirúrgico al existir una intensa reacción desmoplásica/inflamatoria alrededor del tumor que impida separar el tumor del vaso. La experiencia del equipo quirúrgico en este tipo de cirugías y en técnicas de revascularización va a determinar en gran medida que un tumor sea resecable o no. 
Desde un punto de vista práctico, hay que entender que determinar si un tumor pancreático es resecable quirúrgicamente es extremadamente complicado, incluso durante el acto quirúrgico. La combinación de todos los datos y pruebas de imagen que podamos aportar al cirujano antes de la intervención (incluyendo la USE), deberían ayudar a tomar la decisión más correcta para cada paciente. Por desgracia no existe ninguna técnica que por sí sola sea capaz de asegurar la resecabilidad de la lesión.

Conclusión 3. "La USE-PAAF de los tumores del páncreas tiene una elevada precisión diagnóstica (> 90\%) y una reducida tasa de complicaciones $(<1 \%)$ ". Aunque el rendimiento de la USE-PAAF en este tipo de pacientes es elevado (11), no todos los autores aceptan las mismas indicaciones. En nuestra opinión, sólo debería realizarse USE-PAAF de masas pancreáticas si el resultado de la misma puede modificar el manejo clínico del paciente. Las situaciones clínicas que en las que nos parece razonable realizar USE-PAAF son: a) sospecha de que la estirpe histológica del tumor no sea adenocarcinoma ductal pancreático (linfoma, tumor neuroendocrino, metástasis pancreática de otro tumor), ya que las implicaciones terapéuticas y/o pronósticas pueden ser diferentes; b) tumor pancreático irresecable según las técnicas de imagen (no candidato a resección quirúrgica, pero sí a quimioterapia); y c) pacientes en los que la resecabilidad es dudosa y se les va a ofrecer neoadyuvancia previa a la cirugía (12). En nuestra opinión, sería razonable no realizar biopsia de aquellas lesiones pancreáticas compatibles con adenocarcinoma ductal que parezcan resecables en las técnicas de imagen, ya que el resultado de la punción (ya sea positivo o negativo) no va a cambiar el manejo terapéutico del paciente (intento de resección quirúrgica) y por lo tanto estaríamos sometiendo al paciente a un riesgo innecesario (12).

Por otro lado, hay que recordar que la USE ha evolucionado desde su nacimiento hasta convertirse en la actualidad en un importante instrumento terapéutico. La posibilidad de realizar USE-PAAF con ecoendoscopios lineales, dotados con un canal de trabajo terapéutico, ha propiciado el desarrollo de diferentes aplicaciones terapéuticas guiadas por USE: neurolisis del plexo celiaco, drenaje de pseudoquistes pancreáticos y abscesos perirrectales, punción y drenaje de la vía biliar y del páncreas como tratamiento paliativo de tumores, implante de semillas de braquiterapia,... (13). La experiencia en este campo es todavía limitada (no disponible en todos los centros) y tanto su eficacia como su seguridad son por regla general poco conocidas. Este hecho ha propiciado un interés creciente en estos últimos años por realizar experimentos en modelos animales que permitan validar la eficacia y seguridad de este tipo de tratamientos. Un ejemplo claro de esto que acabamos de comentar es el artículo original de López Martín y cols. (6). Los autores de este estudio continúan la línea de investigación iniciada por Fritscher Ravens (14), planteándose investigar en un modelo animal si es posible acceder a las cavidades cardiacas mediante USE-PAAF transesofágica y si este tipo de intervención produce efectos secundarios a nivel cardíaco (arritmias, hemorragia, etc.). Aunque el estudio se realiza en un número reducido de animales, los autores demuestran por primera vez en la literatura que se puede acceder a las cavidades derechas del corazón a través del tabique interauricular, sin evidenciar complicaciones. Sin embargo, las posibles aplicaciones terapéuticas de este nuevo abordaje terapéutico están aún por demostrar. El gran mérito de este estudio reside, en nuestra opinión, en el hecho de que esta es la primera publicación realizada en nuestro medio en la que se utiliza un modelo animal para investigación en ecoendoscopia. Este tipo de estudios, pioneros en nuestro medio, permiten abrir una nueva vía a la investigación dentro del interesante campo de la ecoendoscopia terapéutica. La ampliación del campo de investi- 
gación en ecoendoscopia y su orientación hacia la investigación en modelos animales demuestra que la USE goza de una excelente salud en nuestro medio y que el grado de cualificación de algunos de los grupos de trabajo existentes es muy elevado.

En resumen, la USE es una técnica que está claramente asentada en nuestro sistema sanitario y que por regla general está incluida en el algoritmo preoperatorio de la mayor parte de los tumores digestivos, entre los que destaca el carcinoma de páncreas. El papel relevante de la USE para detectar el tumor, determinar su grado de extensión locorregional y obtener una biopsia es ampliamente aceptado por la comunidad científica. La enorme expansión evidenciada en estos últimos años en el campo de la terapéutica guiada por USE requiere ensayos previos en modelos animales. La aparición de las primeras publicaciones en nuestro medio de estudios de ecoendoscopia terapéutica en modelos animales es ciertamente elogiable y es un fiel reflejo de la buena salud de que goza el mundo de la ecoendoscopia en la actualidad.

E. Vázquez-Sequeiros Servicio de Gastroenterología. Hospital Universitario Ramón y Cajal. Madrid 